\title{
Cancer treatment during COVID-19 pandemic
}

\author{
Vishal Jindal $^{1}$ (D) Kamal Kant Sahu $^{2} \cdot$ Susanna Gaikazian $^{1} \cdot$ Ahmad Daniyal Siddiqui $^{2} \cdot$ Ishmael Jaiyesimi $^{1}$
}

Received: 8 April 2020 / Accepted: 23 May 2020 / Published online: 29 May 2020

(c) Springer Science+Business Media, LLC, part of Springer Nature 2020

\begin{abstract}
Currently world is fighting with global pandemic of coronavirus disease 2019 (COVID-19). At this time of uncertainty, oncologists are struggling to provide appropriate care to cancer patients. They have to weigh risk and benefit of giving cancer treatment vs chances of getting them infected with COVID-19. As cancer patients are immunocompromised and there are high chances of exposure during hospital visits and if they get infected, outcome can be fatal. So through the column of this article, we would like to provide basic guideline in management of cancer patients during COVID-19 pandemic.
\end{abstract}

Keywords COVID-19 Cancer patients $\cdot$ Pandemic

The world is currently fighting against the deadly coronavirus disease 2019 (COVID-19). Globally, the number of people infected and with grave outcomes is increasing exponentially daily [1]. As of 29th April 2020, 2,995,758 people are infected and 204,987 died worldwide [2]. During this time of pandemic, cancer patients are highly susceptible, as they are severely immunocompromised due to both their cancer and its treatment. Many of them are aged and visit medical facilities regularly, which further increases their chance of contact with infected people [3]. Along with this, hospitals are over capacity with COVID-19 patients and most outpatient services are closed to control disease transmission, so it is even more difficult for cancer patients to seek appropriate medical care. This further enhances anxiety and confusion among cancer patients as they deal with the double blow of cancer and COVID-19. Along with cancer patients, oncologists are also facing challenges, as no one has experience with this novel disease and there remain many unanswered questions regarding appropriate care of cancer patients. So through the column of this article, we would like to provide

Vishal Jindal

vishaljindal87@gmail.com; vishal.jindal@beaumont.org

1 Department of Hematology and Oncology, Oakland University-William Beaumont School of Medicine, Beaumont Health, 3601 W. 13 Mile Rd., Royal Oak, MI 48073, USA

2 Hemato-Oncology Division, Department of Internal Medicine, Saint Vincent Hospital, 123 Summer Street, Worcester, MA 01608, USA basic guideline in management of cancer patients during this COVID-19 pandemic.

The major proportion of oncology/hematology clinics consist of treated cancer patients for common tumors such as breast, lung, prostate, or colorectal, as well as hematologic malignancies like non-Hodgkin's lymphoma, multiple myeloma, and chronic lymphoid leukemias. As per Liang et al., patients with cancer have a higher risk of adverse events such as requirement for intensive level of care, mechanical ventilation and death, as compared to non-cancer patients (39\% vs $8 \%, p=0.0003$ ) [4]. These patient groups should be advised to stay indoors and discuss any medical concerns with physicians through telemedicine. Surveillance laboratory testing and imaging should be postponed for several months if a patient is completely asymptomatic. In symptomatic patients, or if there is high suspicion of disease recurrence, physicians should schedule further care based on clinical judgment, considering comorbidities, patient preferences, and tumor biology. A new cancer diagnosis is one of the most distressing times in the life of any patient, as they are dealing with significant uncertainty in understanding their type of cancer, stage, prognosis, treatment options, and much more. At this crucial time, telemedicine can create additional anxiety and confusion and a clinical visit is preferred. This must of course be balanced with the risk of exposure and endemicity of the area.

A major dilemma of oncologists is whether to delay chemotherapeutic treatment or continue it for patients with non-metastatic cancers who are currently on chemotherapy or about to start chemotherapy with curative intent. As per 
Liang et al. [4], patients who have undergone cancer surgery or chemotherapy within the previous 1 month have a higher risk of clinically severe disease as compared to those who did not have surgery or chemotherapy (3/4 [75\%] patients vs. $6 / 14$ [43\%] patients, odds ratio [OR] 5.34, 95\% CI $1.80-16.18 ; p=0.0026)$. Liang suggested the postponement of adjuvant chemotherapy or surgery for stable cancer patients in endemic areas. However, according to another study recently published by Zhang et al. [4], it was recommended that adjuvant cancer chemotherapy should not be withheld or postponed to reduce infection risk in the currently ongoing pandemic. They noted that some patients became infected at infusion centers while receiving chemotherapy, but after weighing risk vs. benefit, concluded that chemotherapy should be continued with extraordinary measures to prevent transmission of COVID-19 disease. Therefore, we recommend that adjuvant chemotherapy for early stage cancer with curative intent should be continued, despite the threat of COVID-19 infection. Since there is a high risk of transmission of infection at infusion centers and all patients are immunocompromised, extreme measures to decrease the spread of COVID-19 should be maintained. Stronger personal protection for patient and their families should be recommended. All patients in chemotherapy infusion areas should be properly screened and low threshold of nucleic acid testing should be considered in suspected patients. If patients present with any signs of active infection, chemotherapy should be deferred and proper quarantine should be maintained. In addition, patients on chemotherapy should be kept in an isolation ward or in at-home isolation for at least 7 days before chemotherapy to prevent administration of any chemotherapy during the possible incubation period of COVID-19.

For patients with metastatic disease who are already on or about to start chemotherapy, strong consideration should be given to overall survival benefit, aggressiveness of cancer, patient performance status, and current levels of COVID19 infection in the area. As Zhang et al. [5] reported in a descriptive analysis, $70 \%$ of stage 4 cancer patients had severe events, and they recommended any aggressive immunosuppressive chemotherapy regime should be dose-reduced or held for patients who are in generally poor condition. However, not providing treatment to stage 4 cancer patients can also increase their hospital admission rate for symptomatic management, which can overwhelm an already heavily burdened medical system. Zhang et al. [5] also showed that $28.6 \%$ of patients developed COVID-19 infection during hospitalization. In another retrospective study, $41.3 \%$ (57/138) of patients acquired COVID-19 during hospitalization, five of these were cancer patients [6]. In addition, treatment delay can lead to worsening of performance status for patients with metastatic disease, and may even close the window for treatment. Therefore, we recommend patients with poor performance status, aggressive disease, and heavy tumor burden should not be considered for chemotherapy, as the risk of contracting COVID-19 supersedes the potential benefit from treatment. Patients with oligometastatic disease, good functional status, and a targetable mutation should be considered for treatment with radiation, surgery, or chemotherapy.

Currently, there is an extensive population of patients on maintenance treatments like Olaparib after chemotherapy for ovarian cancer, rituximab in non-Hodgkin's lymphoma, and many other similar treatments. The challenging question, which is difficult to answer with limited data is, should we stop these immunosuppressive treatments prophylactically as patients are at risk of COVID-19 infection, or should they continue? Our suggestion is to continue treatment with attempts to minimize exposure to COVID-19 as much as possible.

Immune checkpoint inhibitors (ICIs) are one of the major modalities of treatment in advanced cancers. The basis for these treatments is an attempt to enhance the immune system to fight against cancer cells. But how immune modulation will interact with COVID-19 infection and potential treatment is unknown. As it enhances the immune response, immune modulation may provide a benefit in fighting against COVID-19 disease, or it may only enhance the cytokine storm that can lead to severe pneumonitis and respiratory compromise with catastrophic outcomes. Currently, there are no clinical data available, but the risk of a fatal outcome is certainly higher in these patients.

Bone marrow transplants (BMT) that are non-urgent, such as for multiple myeloma patients, should be deferred as much as possible. With the rampant spread of COVID-19, access to bone marrow donors may become limited due to travel restrictions or donor infection. Therefore, we recommend having a frozen stem cell product or alternate donor as back up before the beginning of conditioning treatment. COVID-19 is generally detected in blood; at this point, there are no reports of transmission of disease during transfusions of cellular therapy or blood products. However, any donor diagnosed with COVID-19 must be excluded for donation and deferred for at least 3 months [7]. Considering the current scenario for patients needing urgent transplant, earlier collection should be considered from COVID-19 negative donors. In case of close contact of donors with COVID-19 patients or travel to high risk areas, the donor should be excluded for at least 28 days and monitored for COVID-19 infection [7]. Patients who recently have had BMT should take all precautions to restrict exposure to COVID-19, proper hygiene should be maintained, and patient families should be counseled regarding strict preventive measures to decrease the risk of infection.

As the rampant spread of the COVID-19 pandemic has already overburdened hospitals, a heavy influx of patients, a. 
shortage of ventilators, ICU beds, medical staff, and protective equipment are inevitable [8]. In these circumstances, providing ventilator support to terminal cancer patients will be difficult, as chances of full recovery will be poor. Therefore, proactive palliative and end of life discussions should be held with patients addressing the risk for infection with COVID-19.

In conclusion, it is our duty to provide proper resources and information to patients to help in decision-making at this time of uncertainty. There are still many unanswered questions and we await data from clinical trials to fully answer them. However, we still have the obligation to rise to the challenge of providing the best possible evidence-based management for all cancer patients during this time of crisis and uncertainty.

Author contributions All authors have seen the manuscript and agree to the content and data. All the authors played a significant role in the preparation of this paper.

Funding No funding was received for this study by any entity in the public or private sector.

\section{Compliance with ethical standards}

Conflict of interest Authors have no conflicts of interest to declare.

Ethical approval The article does not contain any regulated human participant or animal research.

\section{References}

1. Sahu KK, Mishra AK, Lal A. Comprehensive update on current outbreak of novel coronavirus infection (2019-nCoV). Ann Transl Med. 2020. https://doi.org/10.21037/atm.2020.02.92.

2. World Heath Organization. Coronavirus disease situation dashboard. https://covid19.who.int/. Accessed 29 Apr 29.

3. Xia Y, Jin R, Zhao J, et al. Risk of COVID-19 for cancer patients. Lancet Oncol. 2020;21(4):PE180. https://doi.org/10.1016/S1470 -2045(20)30150-9.

4. Liang W, Guan W, Chen R, et al. Cancer patients in SARSCoV-2 infection: a nationwide analysis in China. Lancet Oncol. 2020;21(3):P335-337. https://doi.org/10.1016/S1470 -2045(20)30096-6.

5. Zhang L, Zhu F, Xie L, et al. Clinical characteristics of COVID19-infected cancer patients: a retrospective case study in three hospitals within Wuhan, China. Ann Oncol. 2020. https://doi. org/10.1016/j.annonc.2020.03.296. (in press).

6. Wang D, Hu B, Hu C, et al. Clinical characteristics of 138 hospitalized patients with 2019 novel coronavirus-infected pneumonia in Wuhan. China JAMA. 2020;323(11):1061-9. https://doi. org/10.1001/jama.2020.1585.

7. European society of blood and Marrow transplantation guidelines. https://www.ebmt.org/ebmt/news/coronavirus-disease-covid -19-ebmt-recommendations-update-march-23-2020.

8. Ueda M, Martins R, Hendrie P, et al. Managing cancer care during the COVID-19 pandemic: agility and collaboration toward a common goal. J Natl Compr Cancer Netw. 2020. https://doi. org/10.6004/jnccn.2020.7560. (in press).

Publisher's Note Springer Nature remains neutral with regard to jurisdictional claims in published maps and institutional affiliations. 